UDC 53.072; 53:681.3

\author{
A.A. Kupchishin ${ }^{1}$, A.I. Kupchishin ${ }^{2}$, E.V. Shmygalev ${ }^{1 *}$, T.A. Shmygaleva ${ }^{1 *}$, Sh.E. Jeleunova ${ }^{1}$ \\ ${ }^{1}$ Al-Farabi Kazakh National University, Kazakhstan, Almaty \\ ${ }^{2}$ Kazak National Pedagogical University named after Abai, Kazakhstan, Almaty \\ "E-mail: Shmyg1953@mail.ru

\section{Mathematical modelling of radiating formation of defects processes at interoperability of carbon with graphite}

\begin{abstract}
Processes of radiation formation of defects in the carbon irradiated by graphite are considered in work. The regularities arising at selection of approximation expressions, a finding of result area at calculation of cascadely - probabilistic functions depending on number of interactions and depth of penetration of particles are revealed. The regularities formed at calculations of concentration of radiating defects in graphite, irradiated by carbon are received. Results of calculations are presented in the form of tables and schedules.
\end{abstract}

Keywords: modeling, defects, carbon, ions, graphite.

\section{Introduction}

At present, practically there is no general theory of passage of particles through substance. For use of this or that method of calculation it is necessary neatly to define applicability of each method, its possibility, approximation of described interaction, area of applicability of classical methods, quantum concepts, relativistic and non-relativistic physics. Especially it is necessary to note interaction of ions with substance. For the description of radiating formation of defects of ions processes with substance we use an analytical cascadely - probabilistic method (CPM) which essence consists in reception and further use of cascadely - probabilistic functions (CPF).

Table 1 - Approximation parameters for carbon in graphite

\section{Experiment}

CPF calculation for carbon in graphite depending on number of interactions and depth of penetration of particles was done under the formula 6.63 of [1]. For calculation CPF it is necessary to find parameters of approximation $\mathrm{s}_{0}, \mathrm{a}, \mathrm{E}_{0}, \mathrm{k}$. Approximation expression looks like 6.64 [1].

At selection approximation parameters the following regularities take place:

1. With reduction of initial energy of particle the coefficients of approximation $\mathrm{s}_{0}$, a increase, coefficients $\mathrm{E}_{0}, \mathrm{k}$ decrease. The theoretical correlation ratio also decreases (table 1 ).

2 . With reduction of quantity of points the theoretical correlation ratio decreases (table 1).

\begin{tabular}{|c|c|c|c|c|c|}
\hline $\mathrm{E}_{0}, \mathrm{\kappa эB}$ & $\sigma_{0}^{*} 10^{7}$ & $\mathrm{a}$ & $\mathrm{E}_{0}^{\prime}$ & $\mathrm{k}$ & $\eta$ \\
\hline 100 & 2,115 & 8,346 & 0,1 & 110,65 & 0,84 \\
\hline 80 & 2,643 & 10,012 & 0,08 & 106,952 & 0,79 \\
\hline 60 & 3,523 & 12,512 & 0,06 & 102,828 & 0,73 \\
\hline
\end{tabular}

When calculating the CPF depending on number of interactions the following regularities are brought out:

1. With reduction of initial energy at the same depth of observation the internal area of result is narrowed and displaced to the left from $\mathrm{h} / \mathrm{l}$ (table 2).

2. Depending on depth of observation the percent of displacement of the left and right borders of result area decrease. The area of result is displaced to the left from $\mathrm{h} / \mathrm{l}$.

3. Depending on depth of observation the step for calculation increases, on the average changes in a range $8-36$ (table 2 )

4. Depending on initial energy at the same depth of observation the step also increases (table 2). 
Table 2 - Dependence of displacement percent of the left and right borders of result area from number of interactions for carbon in graphite for a) $\mathrm{E}_{0}=100 \mathrm{keV}$; b) $\mathrm{E}_{0}=80 \mathrm{keV}$; c) $\mathrm{E}_{0}=60 \mathrm{keV}$

\begin{tabular}{|c|c|c|c|c|}
\hline $\mathrm{h}^{*} 10^{4}, \mathrm{sm}$ & $\mathrm{B}_{1}$ & $\mathrm{~B}_{2}$ & $\mathrm{~N}_{\mathrm{n}}$ & $\mathrm{B}_{3}$ \\
\hline 1 & 80 & 55 & 8 & 135 \\
\hline 2 & 65 & 5 & 20 & 70 \\
\hline 3 & 63 & -18 & 25 & 28 \\
\hline 4 & 62 & -34 & 30 & 20 \\
\hline 5 & 65 & -45 & 35 & 13 \\
\hline 6 & 68 & -55 & & 28 \\
\hline
\end{tabular}

a)

\begin{tabular}{|c|c|c|c|c|c|}
\hline $\mathrm{h} * 10^{4}, \mathrm{sm}$ & $\mathrm{h} / \mathrm{l}$ & $\mathrm{C}_{1}$ & $\mathrm{C}_{2}$ & $\mathrm{~N}_{\mathrm{h}}$ & $\mathrm{C}_{3}$ \\
\hline 1 & 131 & 26 & 77 & 8 & 103 \\
\hline 2 & 420 & -7 & 56 & 30 & 49 \\
\hline 3 & 971 & -23 & 49 & 50 & 26 \\
\hline 4 & 2011 & $-29,5$ & 43,5 & 100 & 14 \\
\hline
\end{tabular}

b)

\begin{tabular}{|c|c|c|c|c|c|}
\hline $\mathrm{h} * 10^{4}, \mathrm{sm}$ & $\mathrm{h} / \mathrm{l}$ & $\mathrm{C}_{1}$ & $\mathrm{C}_{2}$ & $\mathrm{~N}_{\mathrm{h}}$ & $\mathrm{C}_{3}$ \\
\hline 1 & 241 & 13 & 64 & 13 & 77 \\
\hline 1,5 & 473 & -5 & 53 & 25 & 38 \\
\hline 2 & 817 & -17 & 49,5 & 38 & 32,5 \\
\hline 2,5 & 1324 & -24 & 46,5 & 60 & 22,5 \\
\hline 3 & 2080 & $-28,4$ & 43 & 100 & 14,6 \\
\hline 3,5 & 3244 & $-30,5$ & 39,195 & 155 & 8,695 \\
\hline
\end{tabular}

c)

Here $\mathrm{B}_{1}, \mathrm{~B}_{2}$ are percentages of displacement of the left and right borders of result area, $B_{3}$ is internal result area (in percent), $\mathrm{N}_{\mathrm{n}}$ is a step for calculation.

When calculating the CPF depending on $h$ the following regularities are noted:

1. With reduction of initial energy of a particle at the same depth of observation the percent of internal result area decreases (table 3 ), the result area is displaced to the right from $\mathrm{h} / \mathrm{l}$.

2. With an increase of depth of observation percent of displacement of the left and right borders of result area decrease, the percent of internal re- sult area also decreases and is displaced to the right from $\mathrm{h} / \mathrm{l}$.

3. Depending on depth of supervision the step increases, changes in a range 5-250.

4. CPF calculations depending on number of interactions where the step increases slowly and reaches several tens compared to the step for $\mathrm{CPF}$ calculations depending on depth of penetration rises sharply and reaches several hundreds.

5. Depending on initial energy of a particle at the same depth of observation the step decreases (table 3)

Table 3 - Dependence of displacement percent of the left and right borders of result area from depth of observation for carbon in graphite for a) $\mathrm{E}_{0}=100 \mathrm{keV}$; b) $\mathrm{E}_{0}=80 \mathrm{keV}$; c) $\mathrm{E}_{0}=60 \mathrm{keV}$

\begin{tabular}{|c|c|c|c|c|c|}
\hline $\mathrm{h} * 10^{4}, \mathrm{sm}$ & $\mathrm{h} / \mathrm{l}$ & $\mathrm{C}_{1}$ & $\mathrm{C}_{2}$ & $\mathrm{~N}_{\mathrm{h}}$ & $\mathrm{C}_{3}$ \\
\hline 1 & 82 & 38 & 90 & 5 & 128 \\
\hline 2 & 257 & 1 & 64 & 54 & 65 \\
\hline 3 & 568 & -16 & 54,4 & 32 & 38,4 \\
\hline 4 & 1098 & -26 & 48 & 60 & 22 \\
\hline 5 & 2009 & -30 & 43 & 120 & 13 \\
\hline 6 & 3678 & $-30,5$ & 36 & 250 & 5,5 \\
\hline
\end{tabular}

a) 


\begin{tabular}{|c|c|c|c|c|c|}
\hline $\mathrm{h} * 10^{4}, \mathrm{sm}$ & $\mathrm{h} / \mathrm{l}$ & $\mathrm{C}_{1}$ & $\mathrm{C}_{2}$ & $\mathrm{~N}_{\mathrm{h}}$ & $\mathrm{C}_{3}$ \\
\hline 1 & 131 & 26 & 77 & 8 & 103 \\
\hline 2 & 420 & -7 & 56 & 30 & 49 \\
\hline 3 & 971 & -23 & 49 & 50 & 26 \\
\hline 4 & 2011 & $-29,5$ & 43,5 & 100 & 14 \\
\hline
\end{tabular}

b)

\begin{tabular}{|c|c|c|c|c|c|}
\hline $\mathrm{h}^{*} 10^{4}, \mathrm{sm}$ & $\mathrm{h} / \mathrm{l}$ & $\mathrm{C}_{1}$ & $\mathrm{C}_{2}$ & $\mathrm{~N}_{\mathrm{h}}$ & $\mathrm{C}_{3}$ \\
\hline 1 & 241 & 13 & 64 & 13 & 77 \\
\hline 1,5 & 473 & -5 & 53 & 25 & 38 \\
\hline 2 & 817 & -17 & 49,5 & 38 & 32,5 \\
\hline 2,5 & 1324 & -24 & 46,5 & 60 & 22,5 \\
\hline 3 & 2080 & $-28,4$ & 43 & 100 & 14,6 \\
\hline 3,5 & 3244 & $-30,5$ & 39,195 & 155 & 8,695 \\
\hline
\end{tabular}

c)

Here $\mathrm{C}_{1} \mathrm{C}_{2}$ are percentages of displacement of the left and right borders of result area, $\mathrm{C}_{3}$ is internal result area (in percent), $\mathrm{N}_{h}$ is a step for calculation.

Calculations are lead for carbon in graphite at value of initial energy $\mathrm{E} 0=60,80,100 \mathrm{keV}$.

$\mathrm{CPF}$ behaviour depending on number of interactions is the following:

Curves are crossed among themselves, with an increasing $h$ curves are displaced to the right, the distance between neighbouring curves increases, value of function in a point of a maximum decreases (figure 1).

Depending on depth of penetration at small $\mathrm{n}$ curves are crossed. With an increasing $\mathrm{n}$ the distance between curves increases also curves are not crossed, the internal area of result, value of function in a point of a maximum decrease (figure 2).

Calculation of concentration of radiating defects at an ionic irradiation has allowed to bring out the following regularities:

1. With an increase of threshold energy at the same depth of penetration the values of concentration of radiating defects significantly decreases, borders of result area do not change (table 4).

2. Depending on depth of penetration the values of concentration of radiating defects increase (table 4).

3 . With an increase of initial energy of a primary particle at the same value of threshold energy and depth of penetration the values of concentration of radiating defects decrease.

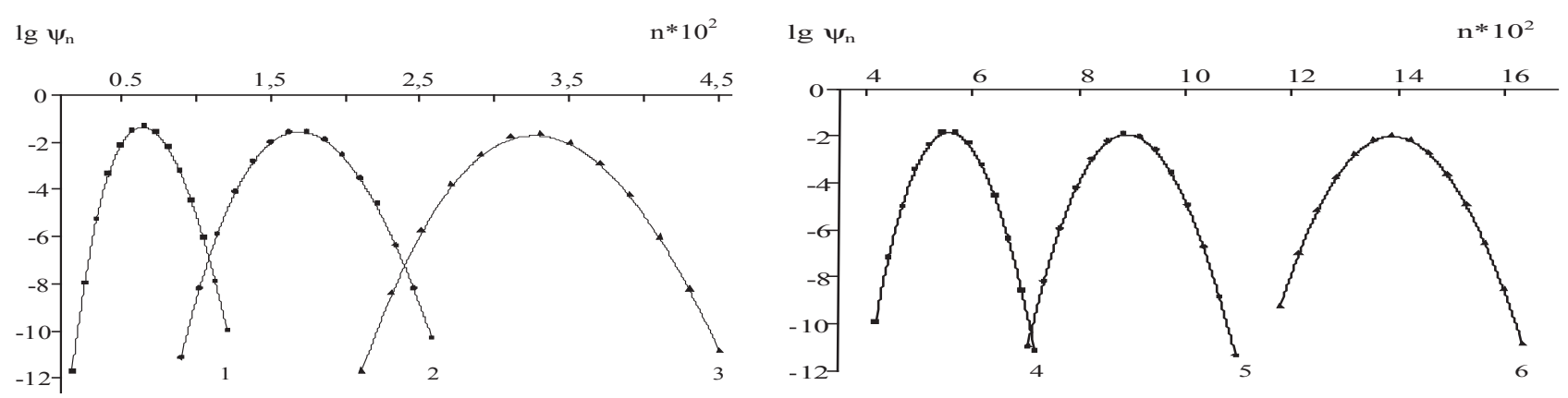

Figure 1 - Dependence $\mathrm{y}_{\mathrm{n}}\left(\mathrm{h}^{\prime}, \mathrm{h}, \mathrm{E}_{0}\right)$ on $\mathrm{n}$ at $\mathrm{E}_{0}=100 \mathrm{keV}$ for $\mathrm{h}=0,0001 ; 0,0002 ; 0,0003 ; 0,0004$; 0,$0005 ; 0,0006 \mathrm{~cm}(1-6)$.

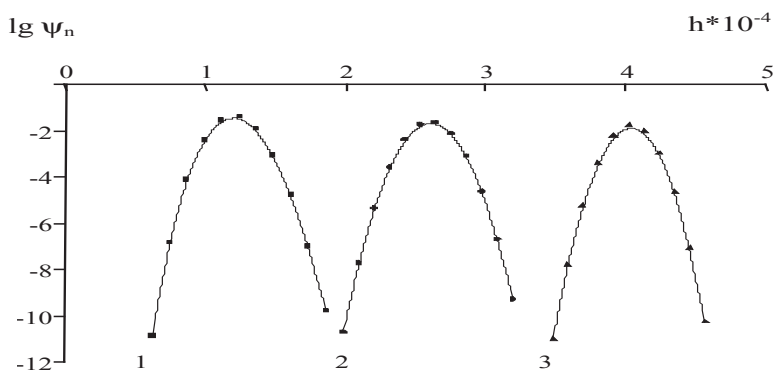

Figure 2 - Dependence $y_{n}\left(h,, h, E_{0}\right)$ on h at $\mathrm{E}_{0}=100 \mathrm{keV}$ for $\mathrm{n}=82,257,568(1-3)$. 


\section{Results and Discussion}

As calculations show (figure 3), with an increase of threshold energy the curves pass significantly lower, transition through a maximum is carried out more smoothly. Concentration of radiating defects depending on depth increases, peaking, then decreases up to 0 .

Table 4 - Borders of a definition range of concentration of radiating defects for carbon in graphite $\mathrm{E}_{\mathrm{c}}=10,15,20 \mathrm{keV}$ at a) $\mathrm{E}_{0}=100 \mathrm{keV}$; b) $\mathrm{E}_{0}=80 \mathrm{keV}$; c) $\mathrm{E}_{0}=60 \mathrm{keV}$

\begin{tabular}{|c|c|c|c|c|}
\hline $\mathrm{h} * 10^{5}, \mathrm{sm}$ & $\mathrm{C}_{\mathrm{k}}$ & $\mathrm{E}_{0}$, кэB & $\mathrm{n}_{0}$ & $\mathrm{n}_{1}$ \\
\hline \multirow[t]{3}{*}{1} & 1783 & \multirow[t]{3}{*}{100} & \multirow[t]{3}{*}{0} & \multirow[t]{3}{*}{21} \\
\hline & 1123 & & & \\
\hline & 793 & & & \\
\hline \multirow[t]{3}{*}{16} & 3773 & \multirow[t]{3}{*}{80} & \multirow[t]{3}{*}{42} & \multirow[t]{3}{*}{183} \\
\hline & 2336 & & & \\
\hline & 1617 & & & \\
\hline \multirow[t]{3}{*}{25} & 5217 & \multirow[t]{3}{*}{70} & \multirow[t]{3}{*}{118} & \multirow[t]{3}{*}{310} \\
\hline & 3188 & & & \\
\hline & 2174 & & & \\
\hline \multirow[t]{3}{*}{33} & 7068 & \multirow[t]{3}{*}{60} & \multirow[t]{3}{*}{240} & \multirow[t]{3}{*}{498} \\
\hline & 4241 & & & \\
\hline & 2827 & & & \\
\hline \multirow[t]{3}{*}{43} & 9510 & \multirow[t]{3}{*}{50} & \multirow[t]{3}{*}{427} & \multirow[t]{3}{*}{759} \\
\hline & 5547 & & & \\
\hline & 3566 & & & \\
\hline \multirow[t]{3}{*}{52} & 12879 & \multirow[t]{3}{*}{40} & \multirow[t]{3}{*}{719} & \multirow[t]{3}{*}{1141} \\
\hline & 7155 & & & \\
\hline & 4293 & & & \\
\hline \multirow[t]{3}{*}{62} & 17718 & \multirow[t]{3}{*}{30} & \multirow[t]{3}{*}{1195} & \multirow[t]{3}{*}{1726} \\
\hline & 8859 & & & \\
\hline & 4429 & & & \\
\hline \multirow[t]{3}{*}{72} & 23453 & \multirow[t]{3}{*}{20} & \multirow[t]{3}{*}{2023} & \multirow[t]{3}{*}{2678} \\
\hline & 7818 & & & \\
\hline & 0 & & & \\
\hline \multirow[t]{2}{*}{83} & 0 & 10 & 3862 & 4776 \\
\hline & 0 & & & \\
\hline
\end{tabular}

a)

\begin{tabular}{|c|c|c|c|c|}
\hline $\mathrm{h} * 10^{5}, \mathrm{sm}$ & $\mathrm{C}_{k}$ & $\mathrm{E}_{0}, \kappa э \mathrm{~B}$ & $\mathrm{n}_{0}$ & $\mathrm{n}_{1}$ \\
\hline \multirow[t]{3}{*}{1} & 2720 & \multirow[t]{3}{*}{80} & \multirow[t]{3}{*}{0} & \multirow[t]{3}{*}{26} \\
\hline & 1684 & & & \\
\hline & 1166 & & & \\
\hline \multirow[t]{3}{*}{8} & 4104 & \multirow[t]{3}{*}{70} & \multirow[t]{3}{*}{18} & \multirow[t]{3}{*}{130} \\
\hline & 2508 & & & \\
\hline & 1710 & & & \\
\hline \multirow[t]{3}{*}{17} & 6097 & \multirow[t]{3}{*}{60} & \multirow[t]{3}{*}{105} & \multirow[t]{3}{*}{294} \\
\hline & 3658 & & & \\
\hline & 2439 & & & \\
\hline \multirow[t]{3}{*}{26} & 8720 & \multirow[t]{3}{*}{50} & \multirow[t]{3}{*}{260} & \multirow[t]{3}{*}{530} \\
\hline & 5087 & & & \\
\hline & 3270 & & & \\
\hline \multirow[t]{3}{*}{36} & 12324 & \multirow[t]{3}{*}{40} & \multirow[t]{3}{*}{527} & \multirow[t]{3}{*}{895} \\
\hline & 6847 & & & \\
\hline & 4108 & & & \\
\hline
\end{tabular}




\begin{tabular}{|c|c|c|c|c|}
\hline \multirow[t]{3}{*}{46} & 17460 & \multirow[t]{3}{*}{30} & \multirow[t]{3}{*}{982} & \multirow[t]{3}{*}{1470} \\
\hline & 8729 & & & \\
\hline & 4365 & & & \\
\hline \multirow{4}{*}{56} & & \multirow{4}{*}{20} & \multirow{4}{*}{1795} & \multirow{4}{*}{2432} \\
\hline & 23453 & & & \\
\hline & 7818 & & & \\
\hline & 0 & & & \\
\hline \multirow[t]{2}{*}{67} & 0 & \multirow[t]{2}{*}{10} & \multirow[t]{2}{*}{3616} & \multirow[t]{2}{*}{4504} \\
\hline & 0 & & & \\
\hline
\end{tabular}

b)

\begin{tabular}{|c|c|c|c|c|}
\hline $\mathrm{h}^{*} 10^{5}, \mathrm{sm}$ & $\mathrm{C}_{k}$ & $\mathrm{E}_{0}$, кэВ & $\mathrm{n}_{0}$ & $\mathrm{n}_{1}$ \\
\hline \multirow[t]{3}{*}{1} & 4624 & \multirow[t]{3}{*}{60} & \multirow[t]{3}{*}{0} & \multirow[t]{3}{*}{40} \\
\hline & 2774 & & & \\
\hline & 1850 & & & \\
\hline \multirow[t]{3}{*}{9} & 7269 & \multirow[t]{3}{*}{50} & \multirow[t]{3}{*}{67} & \multirow[t]{3}{*}{239} \\
\hline & 4240 & & & \\
\hline & 2726 & & & \\
\hline \multirow[t]{3}{*}{19} & 11261 & \multirow[t]{3}{*}{40} & \multirow[t]{3}{*}{280} & \multirow[t]{3}{*}{560} \\
\hline & 6256 & & & \\
\hline & 3754 & & & \\
\hline \multirow[t]{3}{*}{29} & 16818 & \multirow[t]{3}{*}{30} & \multirow[t]{3}{*}{690} & \multirow[t]{3}{*}{1111} \\
\hline & 8409 & & & \\
\hline & 4205 & & & \\
\hline \multirow[t]{3}{*}{39} & 23453 & \multirow[t]{3}{*}{20} & \multirow[t]{3}{*}{1485} & \multirow[t]{3}{*}{2065} \\
\hline & 7818 & & & \\
\hline & 0 & & & \\
\hline \multirow[t]{2}{*}{49} & 0 & \multirow[t]{2}{*}{10} & \multirow[t]{2}{*}{3268} & \multirow[t]{2}{*}{4116} \\
\hline & 0 & & & \\
\hline
\end{tabular}

c)

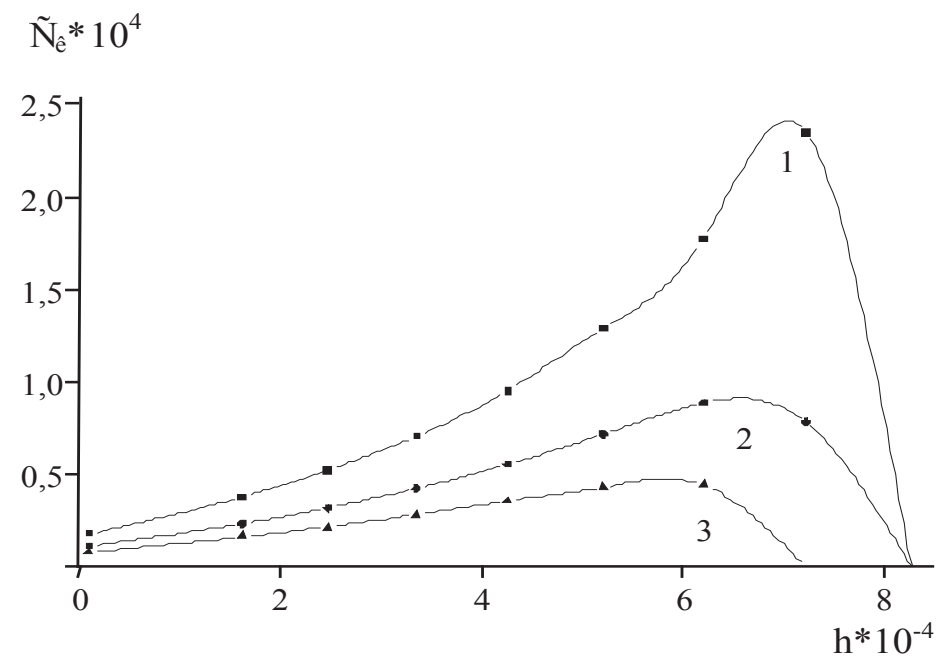

Figure 3 - Dependence $C_{k}\left(E_{0}, h\right)$ from $h$ at $E_{0}=100 \mathrm{keV}$; the $E_{c}=10 ; 15 ; 20 \mathrm{keV}(1-3)$. 


\section{References}

1 Kupchishin A.I., Kylyshkanov M., Shmygaleva T.A., etc. Modelling on the PC and experimental researches of radiating processes in iron and firm alloys. Almaty, Abay KazNAU, Al-Farabi KazNU SRI of ETP, Open Company "KAMA", 2010. - p.263.

А.И. Купчишин, А.А. Купчишин, Т.А. Шмыгалева, Е.В. Шмыгалев, Ш.Е. Джелеунова

Радиациялық міндер пайда болу үдерісіндегі көміртек пен графиттің әсерлесуінде пайда болған математикалық үлгілеулер

Бұл жұмыста графит сәулеге түсірілген көміртек радиациялық білімінің үдерістері қарастырылған. Нәтиже барысында табылған аппроксимация өрнектерін таңдап алып, пайда болатын заңдылықтарды каскадты - ықтимал функциялардың есептеуінде, өзара әрекеттестірген кезде сан бөлшектердің өтімділік тереңдігі айқындалды. Көміртек сәулеге түсірілген графиттегі радиациялық міндердің шоғырландыруына байланысты есептеу құрастыратын заңдылықтар анықталды. Есептеулер қорытындылары графиктер және кестелер түрінде келтірілген.

Tүйін сөздер: модельдеу, ақаулар, көміртек, иондар, графит.

А.И. Купчишин, А.А. Купчишин, Т.А. Шмыгалева, Е.В. Шмыгалев, Ш.Е. Джелеунова Математическое моделирование процессов радиационного дефектообразования при взаимодействии углерода с графитом

В работе рассматриваются процессы радиационного дефектообразования в углероде, облученном графитом. Выявлены закономерности, возникающие при подборе аппроксимационных выражений, нахождении области результата при расчете каскадно-вероятностных функций в зависимости от числа взаимодействий и глубины проникновения частиц. Получены закономерности, образующиеся при расчетах концентрации радиационных дефектов в графите, облученном углеродом. Результаты расчетов представлены в виде графиков и таблиц.

Ключевые слова: моделирование, дефекты, углерод, ионы, графит. 